\title{
The log-linear response function of the bounded number-line task is unrelated to the psychological representation of quantity
}

\author{
Dale J. Cohen $^{1}$ - Philip T. Quinlan ${ }^{2}$
}

Published online: 20 April 2017

(C) Psychonomic Society, Inc. 2017

\begin{abstract}
The bounded number-line task has been used extensively to assess the numerical competence of both children and adults. One consistent finding has been that young children display a logarithmic response function, whereas older children and adults display a more linear response function. Traditionally, these log-linear functions have been interpreted as providing a transparent window onto the nature of the participants' psychological representations of quantity (termed here a direct response strategy). Here we show that the direct response strategy produces the log-linear response function regardless of whether the psychological representation of quantity is compressive or expansive. Simply put, the loglinear response function results from task constraints rather than from the psychological representation of quantities. We also demonstrate that a proportion/subtraction response strategy produces response patterns that almost perfectly correlate with the psychological representation of quantity. We therefore urge researchers not to interpret the $\log$-linear response pattern in terms of numerical representation.
\end{abstract}

Keywords Numerical cognition $\cdot$ Number line $\cdot$ Logarithmic function $\cdot \log -$ linear shift $\cdot$ Computational modeling

Dale J. Cohen and Philip T. Quinlan contributed equally to this work.

Dale J. Cohen

cohend@uncw.edu

1 Department of Psychology, University of North Carolina Wilmington, 601 South College Road, Wilmington, NC 28403-5612, USA

2 University of York, York, UK
According to the National Council of Teachers of Mathematics' (NCTM) Principles and Standards document (Common Core State Standards Initiative, 2010), the link between numerical symbols and one's psychological representation of quantity (termed number sense) is a crucial component in $\mathrm{K}-12$ students' ability to understand symbolic numbers, learn new systems of number representations, understand the relationships among numbers and number systems, and make reasonable estimates. The bounded number-line task is one of the principal tools for teaching and measuring number sense in children (see, e.g., Berteletti, Lucangeli, Piazza, Dehaene, \& Zorzi, 2010; Common Core State Standards Initiative, 2010; Dehaene, Izard, Spelke, \& Pica, 2008; Laski \& Siegler, 2007; Thompson \& Opfer, 2008). Much of the evidence supporting the use of the bounded number-line task to measure number sense has come from studies that have made conclusions about underlying psychological quantity representations from the logarithmic and linear response patterns of children and adults (e.g., Geary, Hoard, Nugent, \& Byrd-Craven, 2008; Laski \& Siegler, 2007; Opfer \& Siegler, 2007; Siegler \& Booth, 2004; Siegler \& Opfer, 2003). In contrast to this well-established view, here we demonstrate that the logarithmic and linear response patterns in the bounded number-line task provide no information about the form of underlying psychological representations of quantity.

The bounded number line task is very simple: A participant is presented with a number together with a horizontal line. The line designates a range of values, with the minimum value situated at the left-most edge of the line and the maximum value situated at the right-most edge. The participant is asked to indicate where on the line the corresponding quantity, conveyed by the number, would be located given the bounded range of values. Of central importance is the shape of the function (henceforth, the response function) relating participants' quantity estimates to the actual target values. Various 
cognitive strategies have been put forward to explain the performance. Traditionally, researchers have assumed that a participant's response function to the bounded number-line task provides a direct window onto the participant's psychological understanding of quantities (Booth \& Siegler, 2006; Opfer \& Siegler, 2012; Opfer, Thompson, \& Kim, 2016; Siegler \& Booth, 2004; we term this a direct response strategy). Researchers who accept the direct response strategy have interpreted the bounded number-line data as indicating that children's psychological understanding of quantities is logarithmic, whereas older children's and adults' psychological understanding of quantities is linear (Booth \& Siegler, 2006; Laski \& Siegler, 2007; Opfer \& Siegler, 2005, 2012; Siegler \& Booth, 2004; Siegler \& Opfer, 2003, Siegler \& Ramani, 2006; Siegler, Thompson, \& Opfer, 2009).

More recently, researchers have extended these ideas by fitting a log-linear function to the data. By introducing systematic changes to the parameters of this function, it can mimic a $\log$ function, a linear function, and a mixture of the two (Anobile, Cicchini, \& Burr, 2012; Opfer, Thompson, \& Kim, 2016). Despite this apparently promising approach, here we show that the constraints of the bounded number-line task force a $\log$-linear response pattern when the participant uses a direct response strategy, regardless of the underlying numerical representation of quantity. We conclude, therefore, that researchers cannot draw any conclusions about participants' representations of quantity on the basis of a log-linear (and thus a logarithmic) response pattern.

There is general agreement in the literature that the psychological understanding of a quantity, say a " 5 ," depends on a representation captured by a distribution on a continuum of quantities (often described as a "mental number line"). Different assumptions have been made about the nature of these quantity distributions and their spacing on the line. Two important theoretical alternatives are (i) the linear model with scalar variability and (ii) the logarithmic model with fixed variability. The linear model with scalar variability assumes that the means of the distributions that represent one's psychological understanding of successive integers (e.g., 1, 2, 3 ) are equidistant from one another but that the standard deviations $(S D \mathrm{~s})$ of those distributions increase proportionally with the quantities they represent. The logarithmic model with fixed variability assumes that the means of the distributions that represent the psychological understanding of successive integers are logarithmically spaced but that the $S D$ s of those distributions remain constant.

The fact that young children produce a logarithmic response pattern in the bounded number-line task sits comfortably with the logarithmic model (Opfer \& Siegler, 2005, 2012; Thompson \& Opfer, 2008). In addition, the fact that older children and adults produce a near linear response pattern on the bounded number-line task sits comfortably with the linear model (Booth \& Siegler, 2006; Siegler \& Booth, 2004). This age-related pattern has led to the hypothesis that there is a developmental change such that an initial logarithmic representational system is replaced by a linear representational system as a person ages. Such a view is captured by the loglinear shift hypothesis (Siegler \& Ramani, 2006; Siegler et al., 2009).

The log-linear shift hypothesis is based on the assumption that the bounded number-line task provides a transparent window on the nature of the mental representation of quantities. It is assumed that a participant's response function will mirror his or her underlying representation of quantity. According to Siegler and Booth (2004), "Several groups of investigators have hypothesized that children's estimation reflects their internal representation of numbers" (p. 429). Furthermore, Opfer et al. (2016) made the argument that the bounded number-line provides an ideal template for directly measuring the underlying quantity representations, in the following way, "Because line length itself is not psychophysically compressive or expansive (Lu \& Dosher, 2013), the task provides a relatively straightforward method for assessing compression in numerical magnitude representations" (p. 12).

Recently, an alternative view has emerged that focuses on the role that strategies play in the bounded number-line task (see, e.g., Barth \& Paladino, 2011; Barth, Slusser, Cohen, \& Paladino, 2011; Chesney \& Matthews, 2013; Cohen \& BlancGoldhammer, 2011; Cohen \& Sarnecka, 2014; Friso-van den Bos et al., 2015; Hurst \& Cordes, 2015; Hurst, Relander, \& Cordes, 2016; Slusser, Santiago, \& Barth, 2013). The counterargument is that (i) successful completion of the bounded number-line task depends on the deployment of an appropriate cognitive strategy, and (ii) only when these strategies are modeled can a meaningful estimate of the participant's underlying quantity representation be obtained. For example, Cohen and Blanc-Goldhammer (2011) presented evidence that adults used a proportion/subtraction strategy to estimate the position of a target value on the bounded number line. The strategy was put forward by Hollands and Dyre (2000) in their work on how observers make proportion estimates. According to Hollands and Dyre, observers estimate the position of the target value from the left-hand boundary as well as from the right-hand boundary (e.g., upper bound - target value). This provides a means by which the observer scales the line to the quantities. This strategy is modeled by the cyclic power model (CPM; see Hollands \& Dyre, 2000). Cohen and BlancGoldhammer found that the CPM provided a fit to adult bounded number-line data that was superior to either the linear or the logarithmic function. These results were replicated in older children (Barth \& Paladino, 2011; Cohen \& Sarnecka, 2014; Slusser et al., 2013).

To date, researchers have assumed that the direct response strategy and the proportion/subtraction strategy give rise to response functions that correlate with the underlying quantity representations. Perhaps surprisingly, 
Table 1 Fit statistics for the log-linear model to data simulated using the direct response strategy with underlying quantity representations having numerical biases ranging from 0.6 (negatively accelerating) to 1.4 (positively accelerating) and varying estimated unit sizes

Underlying Psychological Representation: Distributed Gamma Numerical Bias

$\begin{array}{lllll}0.6 & 0.8 & 1 & 1.2 & 1.4\end{array}$

Unit Size

$\begin{array}{lllllll}0.5 & p(\log ) & 0.52 & 0.25 & 0.28 & 0.61 & 0.87 \\ & R^{2} & .83 & .93 & .95 & .92 & .91 \\ 1 & p(\log ) & 0.46 & 0.21 & 0.22 & 0.60 & 0.95 \\ & R^{2} & .87 & .94 & .95 & .90 & .91 \\ 2 & p(\log ) & 0.41 & 0.29 & 0.20 & 0.59 & 0.91 \\ & R^{2} & .89 & .94 & .96 & .91 & .91 \\ 3 & p(\log ) & 0.55 & 0.24 & 0.25 & 0.57 & 0.91 \\ & R^{2} & .88 & .96 & .94 & .92 & .91\end{array}$

Underlying Psychological Representation: Distributed Normal With Scalar Variability

$\begin{array}{lllllll}0.5 & p(\log ) & 0.44 & 0.19 & 0.02 & 0.0 & 0.43 \\ & R^{2} & .98 & .98 & .99 & .98 & .88 \\ 1 & p(\log ) & 0.46 & 0.21 & 0.12 & 0.61 & 0.97 \\ & R^{2} & .98 & .98 & .99 & .89 & .88 \\ 2 & p(\log ) & 0.49 & 0.27 & 0.81 & 1.0 & 1.0 \\ & R^{2} & .98 & .99 & .90 & .88 & .88 \\ 3 & p(\log ) & 0.47 & 0.7 & 1.0 & 1.0 & 1.0 \\ & R^{2} & .98 & .93 & .84 & .84 & .80\end{array}$

Underlying Psychological Representation: Distributed Normal With Fixed Variability

\begin{tabular}{lllllll}
0.5 & $p(\log )$ & 0.47 & 0.20 & 0.02 & 0.0 & 0.32 \\
& $R^{2}$ & .99 & .99 & .99 & .99 & .93 \\
1 & $p(\log )$ & 0.49 & 0.21 & 0.02 & 0.45 & 0.86 \\
& $R^{2}$ & .99 & .99 & .99 & .93 & .92 \\
2 & $p(\log )$ & 0.48 & 0.19 & 0.60 & 1.0 & 1.0 \\
& $R^{2}$ & .99 & .99 & .93 & .92 & .75 \\
3 & $p(\log )$ & 0.47 & 0.51 & 1.0 & 1.0 & 1.0 \\
& $R^{2}$ & .99 & .96 & .92 & .88 & .76 \\
\hline
\end{tabular}

this assumption has never been tested. As a consequence, we report on a number of computer simulations that tested this critical assumption. The key issue is whether reasonably accurate estimates of underlying quantity representations can be made from data produced according to the direct response hypothesis and, separately, according to the proportion/subtraction hypothesis.

\section{Method}

We ran computer simulations of the direct response hypothesis and the proportion/subtraction hypothesis.
Table 2 Fit statistics for the log-linear model and the one-cycle and two-cycle CPM models to data simulated using the proportion/ subtraction strategy with underlying quantity representations having numerical biases ranging from 0.6 (negatively accelerating) to 1.4 (positively accelerating)

Underlying Psychological Representation: Distributed Gamma

$$
\text { Numerical Bias }
$$

Model

$\begin{array}{lllllll}\text { Log-Linear } & p(\log ) & 0.41 & 0.21 & 0.02 & 0.0 & 0.0 \\ & R^{2} & .95 & .98 & .99 & .99 & .98 \\ \text { 1-Cycle CPM } & \text { Estimated Bias } & 0.57 & 0.73 & 0.96 & 1.11 & 1.32 \\ & R^{2} & .96 & .98 & .99 & .99 & .99 \\ \text { 2-Cycle CPM } & \text { Estimated Bias } & 0.54 & 0.73 & 0.87 & 1.10 & 1.32 \\ & R^{2} & .99 & .99 & .99 & .99 & .99\end{array}$

Underlying Psychological Representation: Distributed Normal With Scalar Variability

\begin{tabular}{lllllll} 
Log-Linear & $p(\log )$ & 0.36 & 0.14 & 0.02 & 0.0 & 0.0 \\
& $R^{2}$ & .98 & .99 & .99 & .99 & .98 \\
1-Cycle CPM & Estimated Bias & 0.60 & 0.80 & 0.98 & 1.18 & 1.35 \\
& $R^{2}$ & .99 & .99 & .99 & .99 & .99 \\
\multirow{2}{*}{ 2-Cycle CPM } & Estimated Bias & 0.57 & 0.77 & 0.98 & 0.16 & 1.37 \\
& $R^{2}$ & .99 & .99 & .99 & .99 & .99
\end{tabular}

Underlying Psychological Representation: Distributed Normal With Fixed Variability

\begin{tabular}{lllllll} 
Log-Linear & $p(\log )$ & 0.35 & 0.15 & 0.01 & 0.0 & 0.0 \\
& $R^{2}$ & .98 & .99 & .99 & .99 & .98 \\
1-Cycle CPM & Estimated Bias & 0.61 & 0.80 & 1.00 & 1.19 & 1.37 \\
& $R^{2}$ & .99 & .99 & .99 & .99 & .99 \\
2-Cycle CPM & Estimated Bias & 0.61 & 0.81 & 1.00 & 1.20 & 1.31 \\
& $R^{2}$ & .99 & .99 & .99 & .99 & .99 \\
\hline
\end{tabular}

\section{Quantity representation}

For all simulations, we first simulated underlying quantity representations for the integers contained within the upper and lower boundaries of the number line. Here we only consider a number line ranging from 0 to 50 , but the results would generalize to any range. For each integer, $\Theta_{i}$, we generated a quantity distribution such that the psychological representation of that integer was set to $\psi_{i}=\Theta^{\beta}{ }_{i}+e_{i}$, where $\beta$ denotes the numerical bias and $e$ denotes the error around the mean. For the simulation, we varied $\beta$ from 0.6 (negatively accelerating, similar to the $\log$ ) to 1.4 (positively accelerating, similar to exponential), in increments of 0.2 . When $\beta=1$, there is no bias. We simulated a variety of error distributions: that is, normal distributions with fixed and scalar variance, and the gamma distribution. We focus on the simulations with a gamma distribution [shape parameter $=\log (\operatorname{target})+1]$. The gamma distribution produces data similar to the normal distribution with scalar variance, but it has the advantage of never 
Numerical Bias

\section{8} 1

1.2

1.4

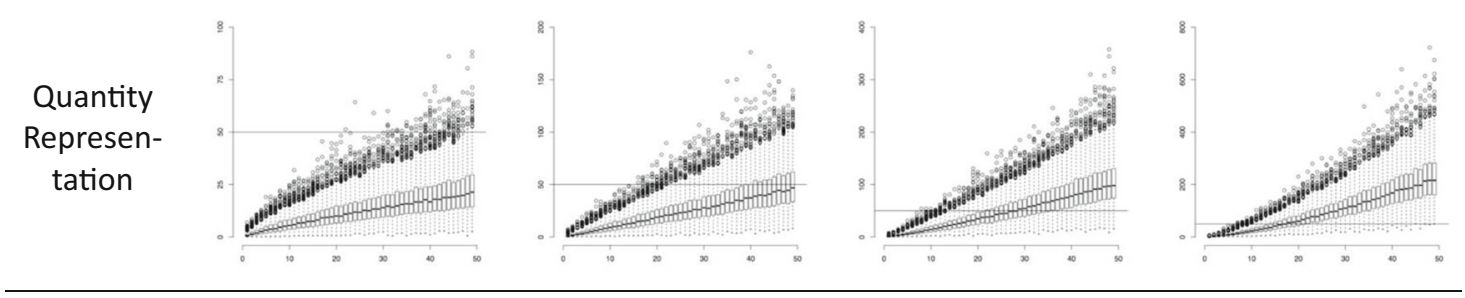

Unit Size

3.0
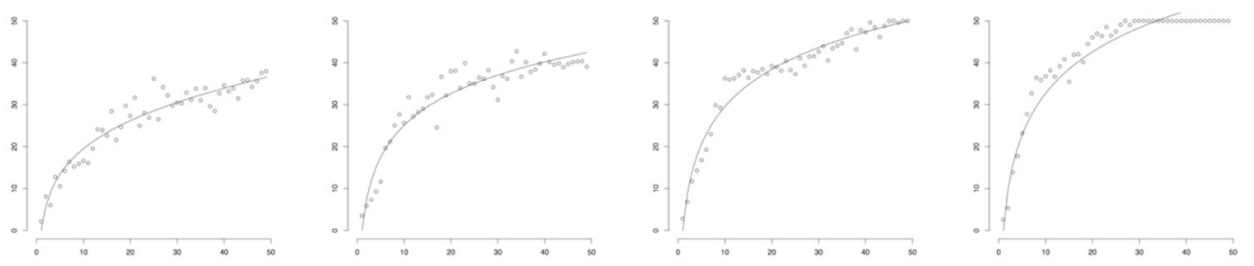

2.5
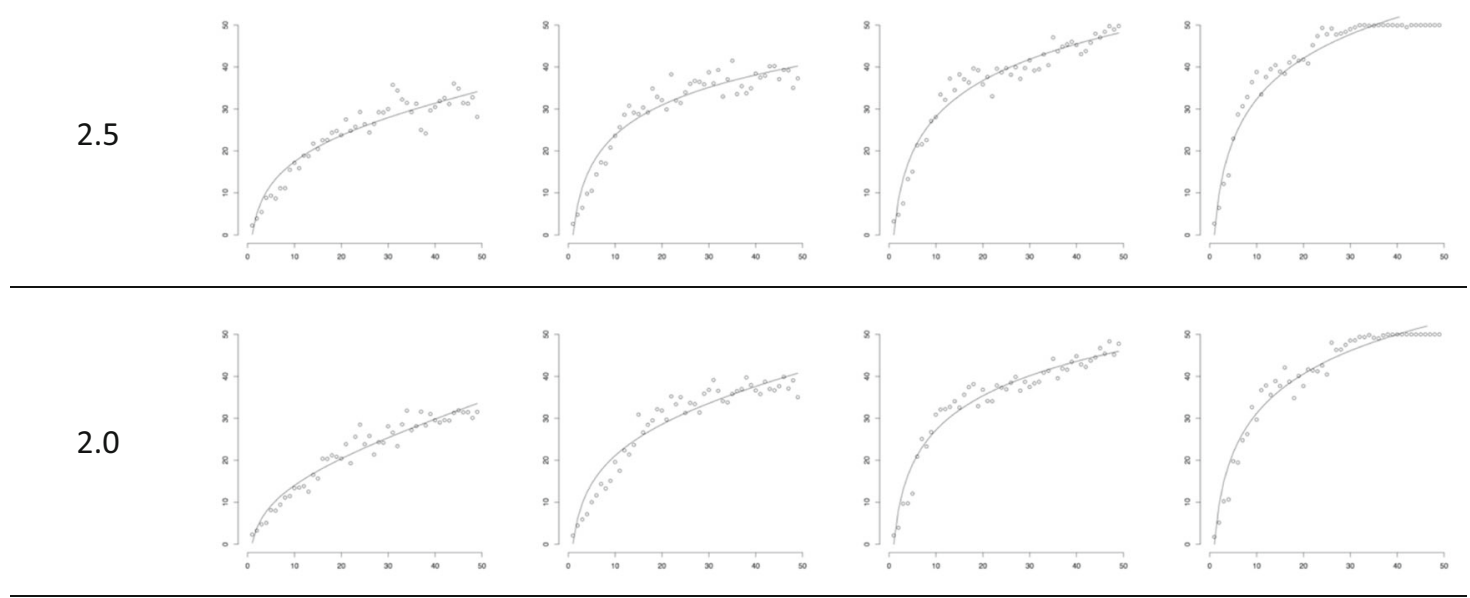

1.5
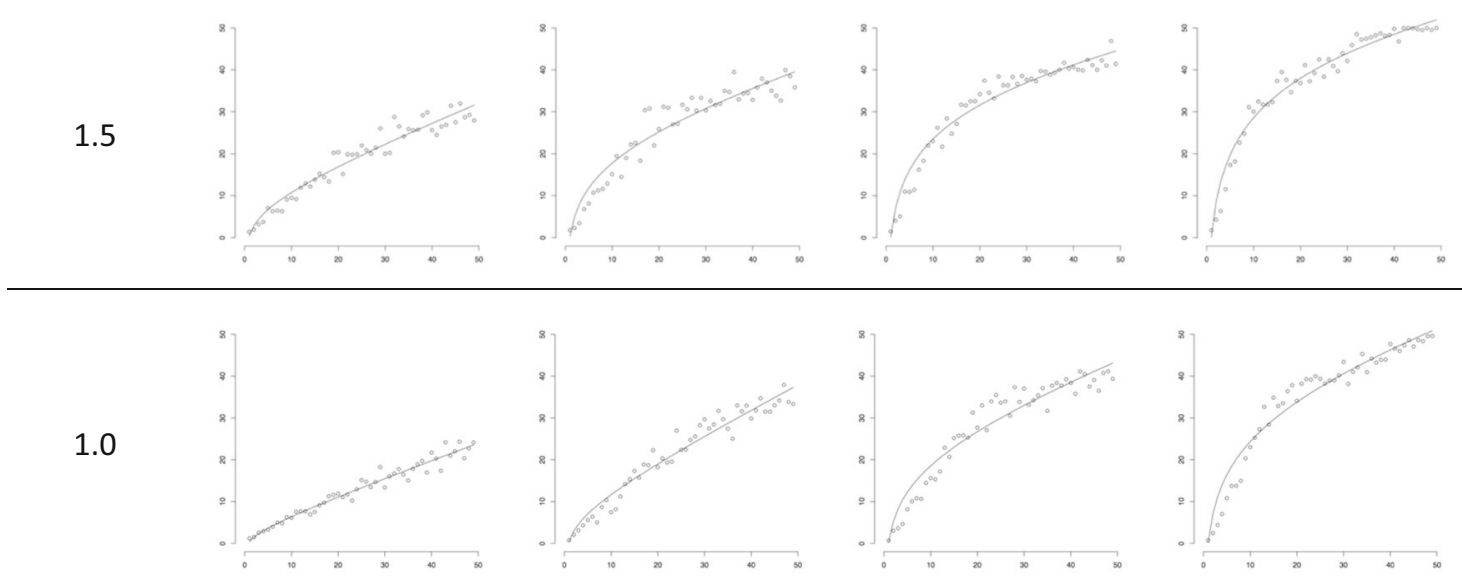

0.5
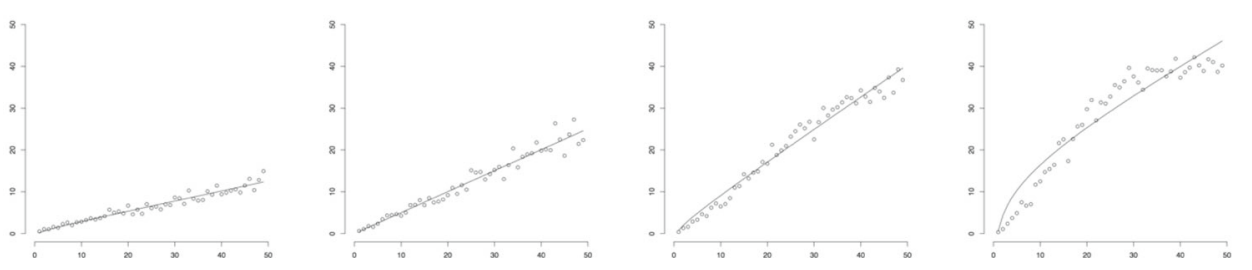
Fig. 1 Simulated underlying gamma quantity representations, their resulting response estimates, and the log-linear fit to the simulated data for the direct response strategy in the bounded number-line task. The columns represent changes in numerical bias (ranging from 0.8 to 1.4, in increments of 0.2 ), and the rows represent changes in the estimated line length corresponding to a single unit: Unit size $=1$ is an accurate estimate, unit size $<1$ is an underestimate, and unit size $>1$ is an overestimate producing a value below zero. Any variation in the outcomes was mostly attributable to the chosen standard deviation.

\section{Task completion}

Direct response strategy For each target value, we sampled an estimate from the corresponding quantity distribution. Because the direct response strategy does not specify a means

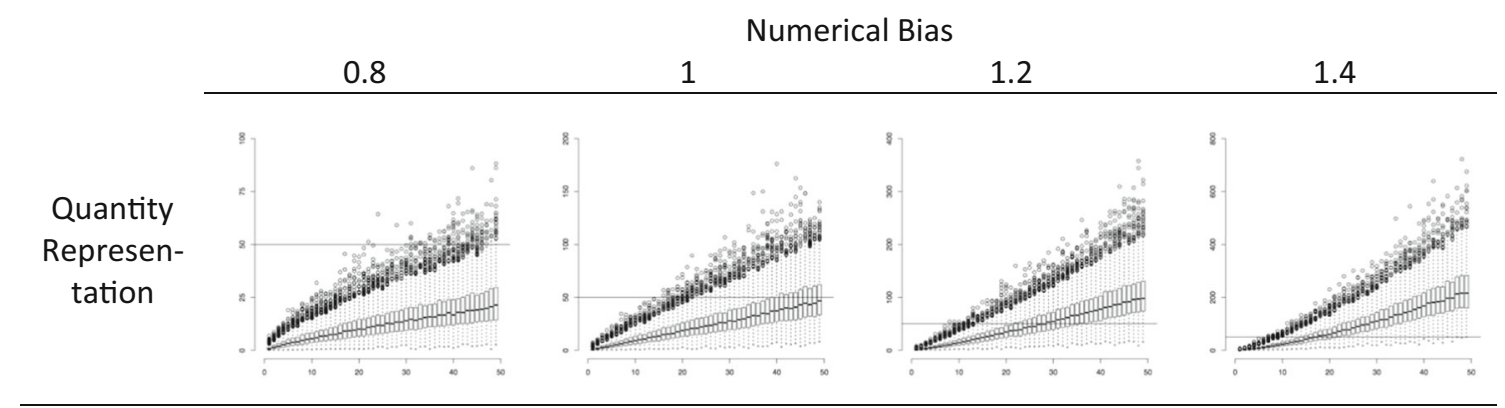

Means

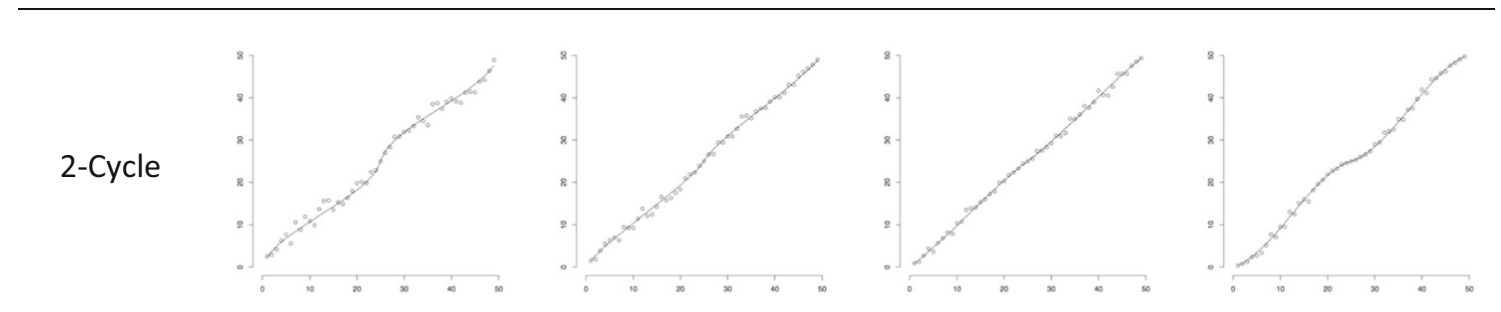

SDs
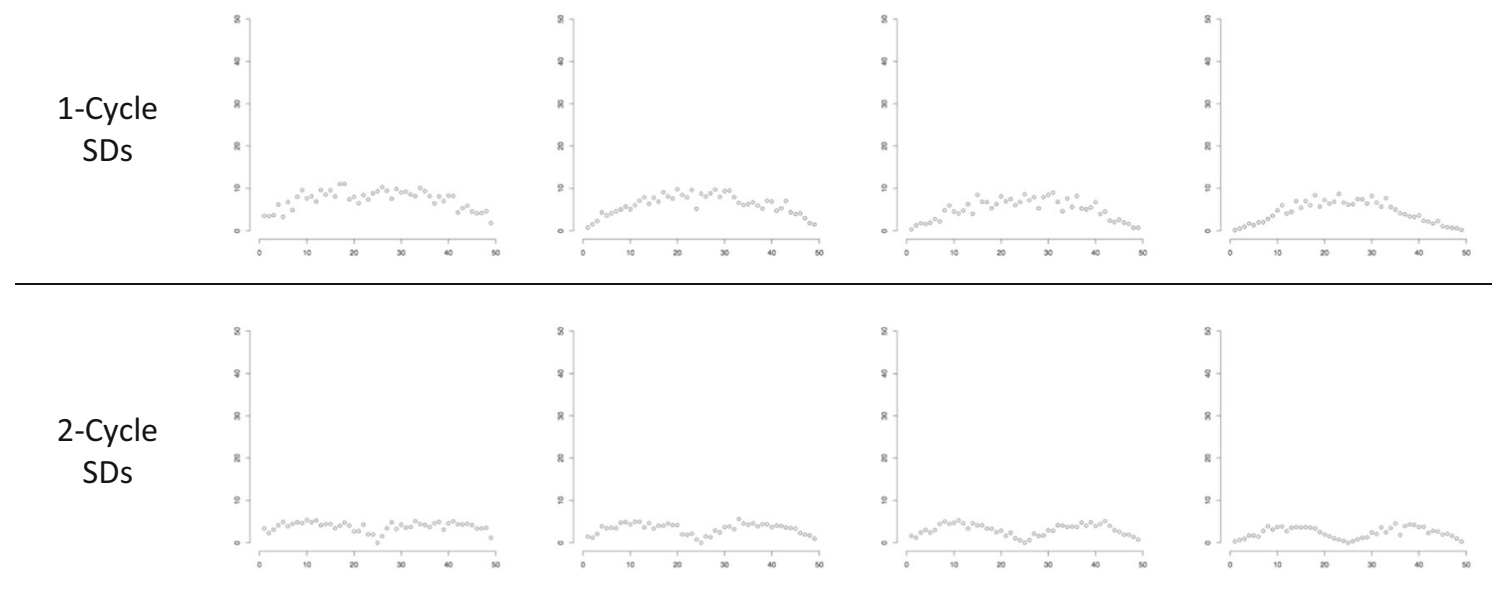

Fig. 2 Simulated underlying gamma quantity representations, their resulting response estimates and $S D$ s, and the one- and two-cycle fits to the simulated data for the proportion/subtraction strategy in the bounded number-line task. The columns represent changes in numerical bias (ranging from 0.8 to 1.4 , in increments of 0.2 ) 
by which an observer maps the length of the line to quantity, the response estimate equaled the sampled estimate multiplied by a unit length. We varied the unit lengths from 0.5 to 3.0 in increments of 0.5 . A unit length of 1.0 indicates an accurate mapping of line length to quantity. The response estimate was valid if it fell between the lower and upper limits of the bounded number line, and invalid if it fell outside these limits. We repeated the sampling and calculation process until we obtained a valid estimate. If we failed to calculate a valid response estimate in 1,000 attempts, we assigned the response estimate the value of the boundary it was closest to. We repeated this process 20 times for each target value.

Proportion/subtraction strategy For each target value, we sampled an estimate from its corresponding quantity distribution and one from the quantity distribution corresponding to the distance from the right boundary (upper boundary - target). We then calculated a response estimate from either the one-cycle or the two-cycle CPM from those values. We repeated the sampling-and-calculation process until we obtained a valid estimate. If we failed to obtain a valid estimate in 1,000 attempts, we assigned the response estimate the value of the boundary it was closest to. We repeated this process 20 times for each target value.

\section{Results}

For each strategy (direct response, one-cycle proportion/subtraction, and two-cycle proportion/subtraction), we fit the loglinear function developed by Anobile et al. (2012; see also Cicchini, Anobile, \& Burr, 2014). The log-linear function has two free parameters: (i) $p(\log )$, which identifies the proportion of the log fit (vs. a linear fit), and (ii) $a$, a scaling parameter. For the one-cycle proportion/subtraction and twocycle proportion/subtraction strategies, we also fit the oneand two-cycle CPMs. The CPM functions have one free parameter: $\beta$, which is the estimate of numerical bias. We present the fit statistics in Tables 1 and 2. We present figures of the fits for a subset of the simulations assuming gamma distributions in Figs. 1 and 2.

As can be seen in Table 1 and Fig. 1, the log-linear function fit all direct response simulations well, regardless of the bias of the underlying quantity representation. In fact, when the bias of the underlying quantity representation is negatively accelerating (similar to a log function), the log-linear function fit least well ( $\beta=0.6$ condition). More concerning is the fact that the $\log$-linear function fit extremely well (mean $R^{2}=.89$ ) for all conditions in which the underlying quantity representation was positively accelerating (expansive, rather than compressive). In addition, $p(\log )$ increases with the positively accelerating bias. These findings result from the fact that observers cannot respond outside the bounds of the bounded number line. As such, the bounds of the number-line truncate the distributions of the larger numbers, thus forcing a negatively accelerating response function. We therefore concluded that the direct response strategy in the bounded number-line task manifests as a log-linear response pattern regardless of the underlying psychological representation of quantity. The clear implication is that the log-linear pattern observed in behavioral data arises because of the constraints of the bounded number-line task, rather than because of the participant's psychological understanding of numbers.

As can be seen in Table 2 and Fig. 2, the one- and two-cycle functions fit all proportion/subtraction response simulations well (mean $R^{2}=.986$ ), regardless of the bias of the underlying quantity representation. Importantly, the estimated $\beta$ s from both the one- and the two-cycle CPM functions are almost perfectly correlated with the simulated $\beta$ s of the quantity representations $(r>.99)$, though with a small but consistent underestimation. Finally, Fig. 2 shows the typical "M"-shaped error pattern that emerges when participants use the halfway mark as a reference point in the bounded number-line task. One can conclude from these data that the proportion/ subtraction response strategy in the bounded number-line task manifests as a CPM response pattern that reflects the response bias, regardless of the underlying psychological representation of quantity.

Finally, we also note that the $\log$-linear function fit all proportion/subtraction response simulations well (mean $R^{2}>$ .98), despite the fact that they were generated using the proportion/subtraction response strategy. This is because the proportion/subtraction response strategy produces relatively linear response patterns with slight curves that the log-linear function can mimic. This simulation underscores Hollands and Dyre's (2000) caution that one must take extreme care not to confuse the linear (or log-linear) function for the CPM.

\section{Discussion}

The results of the simulations are very clear: Log-linear (and thus logarithmic) response patterns in the bounded numberline task provide no information about the underlying quantity representation of the observer. Log-linear (and thus logarithmic) response functions result from the constraints of the task itself: The bounds of the number line truncate the distributions of the larger numbers. In contrast, if participants are able to carry out the proportion/subtraction strategy, the resulting response functions, when modeled correctly, are near perfectly correlated with the numerical bias of the underlying quantity representation.

These findings raise the possibility that the log-linear pattern displayed by young children is the result of a naïve direct response strategy. That is, very young children may not have the mensuration skills to equate a line length with quantity in 
the bounded number-line task (see Cohen \& Sarnecka, 2014). Nevertheless, these young children may still understand the ordinal and interval properties of numbers. In this instance, the children may arbitrarily choose a certain length to correspond to a single unit. The young child may then use this line length to complete the task as we describe in the direct response strategy. In this scenario, the children's responses would necessarily result in a log-linear response function, regardless of the children's underlying psychological representations of quantity.

It is, of course, possible to generate a specific quantity representation or a participant strategy in which our conclusions do not hold. ${ }^{1}$ For example, a quantity representation that is both noise-free and somehow scaled to the dimensions of the number line will be mirrored in a participant's responses with a version of the direct response strategy. The ability to generate such an example, however, does not weaken our conclusions as they currently stand. The strength of a scientific model is typically gauged relative to the number of alternative hypotheses that can also explain the data. Here we demonstrated that when the log-linear function fits well with number-line data, this result alone is consistent with innumerable alternative models that explain that fit (e.g., different underlying quantity representations and strategies). As a result, the log-linear fit provides no substantive evidence about the underlying psychological representation of quantities. This result reflects a fatal flaw in the many previous studies that have used such fits to support particular theoretical accounts of numerical competency. In contrast, when the CPM function fits the number-line data, currently the only model that explains this fit is the proportion/subtraction strategy applied on a specific underlying quantity representation. Until researchers propose viable alternative models that predict the CPM fits as well as the proportion/subtraction strategy model does, the most plausible conclusion is that the CPM provides important information about the underlying psychological representation of the quantity.

In sum, we assert, on the basis of our computer simulations, that the bounded number-line task does not provide a transparent window onto the mental representation of quantities. The constraints of the task produce a log-linear response function when the direct response strategy is applied, regardless of the underlying psychological representation of quantity. We therefore urge that researchers stop offering psychological accounts of the log-linear response functions (and, hence, of logarithmic or linear response functions, or a mixture of the two) in the bounded number-line task that are based on

\footnotetext{
${ }^{1}$ One may question our simulation of negatively accelerating quantity representations using power functions, rather than a log function. Here we demonstrated that the log-linear function fits the responses derived from all quantity representations, regardless of their form (positively or negatively accelerating). Demonstrating that the log-linear function also fits the responses manifesting from a true logarithmic quantity representation is unnecessary.
}

particular assumptions about the mental representation of quantities. We temper this negative conclusion with a more positive encouragement: If the proportion/subtraction strategy is deployed by the participant and modeled by the researcher, then one can recover useful information about the participant's quantity representation. Furthermore, researchers may also consider using the unbounded number-line task (see Cohen \& Blanc-Goldhammer, 2011). This avoids the problems we have described, and critically, the associated data are uncontaminated by the effects of truncation that are evident in those from the bounded number-line task.

\section{References}

Anobile, G., Cicchini, G. M., \& Burr, D. C. (2012). Linear mapping of numbers onto space requires attention. Cognition, 122, 454-459. doi:10.1016/j.cognition.2011.11.006

Barth, H. C., \& Paladino, A. M. (2011). The development of numerical estimation: Evidence against a representational shift. Developmental Science, 14, 545-551. doi:10.1111/j.1467-7687.2010.00962.x

Barth, H. C., Slusser, E. B., Cohen, D., \& Paladino, A. M. (2011). A sense of proportion: Commentary on Opfer, Siegler and Young. Developmental Science, 14, 1205-1206. doi:10.1111/j.1467-7687. 2011.01081.x

Berteletti, I., Lucangeli, D., Piazza, M., Dehaene, S., \& Zorzi, M. (2010). Numerical estimation in preschoolers. Developmental Psychology, 46, 545-551. doi:10.1037/a0017887

Booth, J. L., \& Siegler, R. S. (2006). Developmental and individual differences in pure number estimation. Developmental Psychology, 41, 189-201. doi:10.1037/0012-1649.41.6.189

Chesney, D. L., \& Matthews, P. G. (2013). Knowledge on the line: Manipulating beliefs about the magnitudes of symbolic affects the linearity of line estimation tasks. Psychonomic Bulletin \& Review, 20, 1146-1153. doi:10.3758/s13423-013-0446-8

Cicchini, G. M., Anobile, G., \& Burr, D. C. (2014). Compressive mapping of number to space reflects dynamic encoding mechanisms, not static logarithmic transform. Proceedings of the National Academy of Sciences, 111, 7867-7872. doi:10.1073/pnas.1402785111

Cohen, D. J., \& Blanc-Goldhammer, D. (2011). Numerical bias in bounded and unbounded number line tasks. Psychonomic Bulletin \& Review, 18, 331-338. doi:10.3758/s13423-011-0059-Z

Cohen, D. J., \& Sarnecka, B. (2014). Children's number-line estimation shows development of measurement skills (not number representations). Developmental Psychology, 50, 1640-1652. doi:10.1037/ a0035901

Common Core State Standards Initiative. (2010). Retrieved from www. corestandards.org/Math/

Dehaene, S., Izard, V., Spelke, E., \& Pica, P. (2008). Log or linear? Distinct intuitions of the number scale in Western and Amazonian indigene cultures. Science, 320, 1217-1220. doi:10.1126/science. 1156540

Friso-van den Bos, I., Kroesbergen, E. H., Van Luit, J. E. H., XenidouDervou, I., Jonkman, L. M., Van der Schoot, M., \& Van Lieshout, E. C. D. M. (2015). Longitudinal development of number line estimation and mathematics performance in primary school children. Journal of Experimental Child Psychology, 134, 12-29. doi:10. 1016/j.jecp.2015.02.002

Geary, D., Hoard, M. K., Nugent, L., \& Byrd-Craven, J. (2008). Development of number line representations in children with 
mathematical learning disability. Developmental Neurobiology, 33, 277-299. doi:10.1080/87565640801982361

Hollands, J. G., \& Dyre, B. P. (2000). Bias in proportion judgments: The cyclical power model. Psychological Review, 107, 500-524. doi:10. 1037/0033-295X.107.3.500

Hurst, M., \& Cordes, S. (2015). Rational number comparisons across notation: Fractions, decimals, and whole numbers. Journal of Experimental Psychology: Human Perception and Performance, 42, 281-293. doi: $10.1037 / x h p 0000140$

Hurst, M., Relander, C., \& Cordes, S. (2016). Biases and benefits of number lines and pie charts in proportion representation. In A. Papafragou, D. Grodner, D. Mirman, \& J. C. Trueswell (Eds.), Proceedings of the 38th Annual Conference of the Cognitive Science Society (pp. 586591). Austin, TX: Cognitive Science Society.

Laski, E. V., \& Siegler, R. S. (2007). Is 27 a big number? Correlational and causal connections among numerical categorization, number line estimation, and numerical magnitude comparison. Child Development, 78, 1723-1743.

Lu, Z., \& Dosher, B. A. (2013). Visual psychophysics: From laboratory to theory. Cambridge, MA: MIT Press.

Opfer, J. E., \& Siegler, R. S. (2005). Microgenetic changes in representations of numerical magnitude. In B. G. Bara, L. Barsalou, \& M. Bucciarelli (Eds.), Proceedings of the XXVII Annual Conference of the Cognitive Science Society (pp. 1684-1689). Mahwah, NJ: Erlbaum.

Opfer, J. E., \& Siegler, R. S. (2007). Representational change and children's numerical estimation. Cognitive Psychology, 55, 169-195. doi:10.1016/j.cogpsych.2006.09.002
Opfer, J. E., \& Siegler, R. S. (2012). Development of quantitative thinking. In K. J. Holyoak \& R. G. Morrison (Eds.), Oxford handbook of thinking and reasoning (pp. 1684-1689). Oxford, UK: Oxford University Press.

Opfer, J. E., Thompson, C. A., \& Kim, D. (2016). Free versus anchored numerical estimation: A unified approach. Cognition, 149, 11-17. doi:10.1016/j.cognition.2015.11.015

Siegler, R. S., \& Booth, J. L. (2004). Development of numerical estimation in young children. Child Development, 75, 428-444. doi:10. $1111 / \mathrm{j} .1467-8624.2004 .00684 . x$

Siegler, R. S., \& Opfer, J. E. (2003). The development of numerical estimation: Evidence for multiple representations of numerical quantity. Psychological Science, 14, 237-243. doi:10.1111/14679280.02438

Siegler, R. S., \& Ramani, G. B. (2006). Early development of estimation skills. APS Observer, 19, $34-44$.

Siegler, R. S., Thompson, C. A., \& Opfer, J. E. (2009). The logarithmicto-linear shift: One learning sequence, many tasks, many time scales. Mind, Brain and Education, 3, 143-150. doi:10.1111/j. 1751-228X.2009.01064.X

Slusser, E. B., Santiago, R. T., \& Barth, H. C. (2013). Developmental change in numerical estimation. Journal of Experimental Psychology: General, 142, 193-208. doi:10.1037/a0028560

Thompson, C. A., \& Opfer, J. E. (2008). Costs and benefits of representational change: Effects if context on age and sex differences in symbolic magnitude estimation. Journal of Experimental Child Psychology, 101, 20-51. doi:10.1016/j.jecp.2008.02.003 